\title{
The Analysis of Asymetric Dimethylarginine and Homocysteine in Patients with Chronic Kidney Disease
}

\author{
Tetty Hendrawati ${ }^{1,2}$, Syakib Bakri ${ }^{3}$, Mansyur Arif ${ }^{3}$
}

$\mathrm{B}$

ACKGROUND:Asymmetric dimethylarginine (ADMA) is a competitive inhibitor of nitric oxide synthase (NOS). ADMA reduces NO synthesis when its concentration elevates. ADMA is a novel risk factor for cardiovascular disease. Plasma ADMA accumulates in patients with endstage renal disease, due to reduced renal clearance. Hyperhomocysteinemia is often found in patients with chronickidney disease(CKD).Homocysteinemaycause ADMA to accumulate; however, the mechanism by which ADMA level elevates in hyperhomocysteinemia is still unclear. Objective of this study was to analyze the concentrations of homocysteine and ADMA and to assess the correlation between homocysteine and ADMA concentrations with the severity of chronic kidney disease.

METHODS: This was a cross-sectional study on 75 patients with CKD, comprising men and women aged 40-70 years. Assessments were done on the concentrations of creatinine, homocysteine, ADMA, fasting blood glucose, cholesterol HDL and triglyceride.

RESULTS: In later stage of CKD there was significantly higher tHcy concentration as compared with the earlier stage of CKD ( $p=0.0000)$. In CKD stage 2 to 4 there was a tendency for ADMA concentration to increase to a significant average $(\mathrm{p}=0.210)$, but ADMA concentration was lower at stage 5. There was increased ADMA along with increased tHcy concentration of around $20 \mu \mathrm{mol} / \mathrm{L}$, and this then decreased. The inverse correlation between tHcy and ADMA concentrations started to appear in CKD stage 4, but this correlation was statistically insignificant $(\mathrm{r} 2=0.19 ; \mathrm{p}=0.499)$.
CONCLUSIONS: This study showed there was a correlation between homocysteine and ADMA concentrations in patients with CKD stage 2 to 5 , although statistically not significant.

KEYWORDS: Asymetric Dimethylarginine, Homocysteine, Chronic Kidney Disease.

\section{Introduction}

Kidney failure (stage 5 Chronic Kidney Disease = CKD) is an important health problem, considering its increasingly high incidence and prevalence besides the very expensive medication, including renal replacement therapy, that the patients with kidney failure must undergo. Globally, in 1966 there were approximately one million patients with CKD underwent renal replacement therapy (hemodialysis, peritoneal dialysis, or kidney transplantation); and this number will increase to 2 million in 2010 (1).

Cardiovascular disease is often seen in patients with CKD either at the pre-dialysis phase or mainly at the dialysis phase. Cardiovascular disease is the main cause of death in patients with CKD, with its estimated incidence 10 to 100 times greater than that in the population without CKD (2).

In addition to the conventional/traditional factors of cardiovascular disease such as smoking, diabetes, hypertension, dyslipidemia, and obesity at all stages of kidney disease, there are non-traditional risk factors or uremia-related risk factors such as

\footnotetext{
${ }^{1}$ Prodia Clinical Laboratory, Jakarta

2 Post Graduate Program in Clinical Biochemistry, Hasanuddin University, Makassar

${ }^{3}$ Faculty of Medicine, Hasanuddin University, Makassar
} 
increased homosysteine, C-reactive protein (CRP), asymmetric dimethylarginine (ADMA) concentration, occurrence of microinflammation, proteinuria, and increased oxidative stress, all of which contribute to the progression of these cardiovascular disease risks $(2,3)$.

Clinical evidences have indicated that not all patients with CKD in the early stage progressed into renal failure, and that the percentage of early stage CKD that progressed to renal failure varied among patients. It was suggested that several factors contributed to the progression of early stage chronic kidney disease into kidney failure, and intervention on the risk factors if done at the early stage could slow down progression; in fact in several conditions it could even totally stop progression into kidney failure (1).

Homocysteine is an amino acid-containing sulphydril group that originates from the essential amino acid methionin that comes in great abundance from vegetable fat. The metabolic pathway that changes methionine to homosysteine is very important for many biomolecules to maintain their normal functions, including DNA, proteins, phospholipids and neurotransmitters (4). Homocysteine concentration elevates along with worsening of the kidney function $(2,5)$. In several studies homocysteine was proposed as a cardiovascular risk factor.

Asymmetric dimethylarginine (ADMA) is an arginine form modified post-translationally from all cells during protein modification process. ADMA is a guanidine compound synthesized in endothelial cells. ADMA concentration elevates along with the worsening of the kidney function (6). ADMA is a competitive inhibitor from endothelial nitric oxide synthase (eNOS). eNOS is an enzyme that modifies L-arginine to become nitric oxide (NO). NO plays an important role in maintaining normal endothelial functions as vasodilator, anti thrombosis, and anti proliferation. Elevated ADMA can diminish NO availability, thus ADMA is suggested to be an important cardiovascular risk factor (7).

Several studies using experimental animals and clinical studies have indicated there was a close correlation between total plasma homocycsteine and plasma ADMA concentration (8).

Homocysteine is an important part of methylation process. S-adenosylomethionine (SAM) is a methyl donor that enables arginine methylation and produce S-adenosylhomocysteine (SAH); in turn it can be modified into homocysteine. Results of several studies suggested that ADMA produced by human endothelial cells was upregulated in the presence of methionine or homocysteine and inhibited by metyltransferaseinhibiting SAH.

In situations where homocysteine level increases, the concentration of intracellular $\mathrm{SAH}$ can elevate. Since SAH is a potent inhibitor to transmethylation reactions, its accumulation can cause macromolecules hypomethylation. According to this mechanism, DNA hypomethylation occurs in hyperhomocysteinemic patients with end-stage kidney disease. In the later condition, production of ADMA by endothelial cells is inhibited through incubation by SAH (9).

Two isoforms of dimethylarginine dimethylhidrolase (DDAH) have been identified: DDAH-I is mostly found in the neural tissues, whereas DDAH-II dominates in peripheral and vascular tissues. DDAH is an intracellular protein whose role in several disease conditions has been studied. Because its enzymatic role is to selectively hydrolyze ADMA to form L-citrulline and dymetilamine, reduced enzymatic DDAH expression or activity can elevate the concentration of ADMA (9).

Homocysteine can inhibit DDAH activity, probably because of its interaction with important cysteine residue in active enzyme site. Several studies have suggested the possibility that every existing correlation between homocysteine and cardiovascular risk might possibly indicate a correlation reflection between ADMA and cardiovascular risk (6).

\section{Methods}

\section{STUDY DESIGN AND PARTICIPANTS}

This study used a Cross Sectional Study design. The study population comprised patients examined at the Kidney Polyclinic of Wahidin Hospital, Makasar. The study subjects were selected from the study population who qualified as samples according to the inclusion and exclusion criteria. Inclusion criteria for samples are : (1) Suffering chronic kidney disease according to the criteria of Kidney Disease Quality Outcome Initiative (K/DOQI), (2) Suffering diabetes mellitus according to PERKENI criteria, (3) Not undertaking hemodyalisis, (4) Not undertaking medication with Statin derivates, and Exclusion criteria for sample is subjects who rejected to participate in the study. 


\section{DATA COLLECTION}

The examinations $\mathrm{w}$ ere conducted a $\mathrm{t}$ the $\mathrm{K}$ idney Polyclinic o f Wahidin Hospital, Makasar, $\mathrm{P}$ rodia Clinical Laboratory Branch of Makassar, and Prodia Clinical Laboratory Kramat-Jakarta, from November 2006 to March 2007. Sampling was done by consecutive sampling method, i.e. selecting samples from patients visiting the Kidney Polyclinic of Wahidin Hospital, Makasar throughout the study period who met the inclusion criteria, $\mathrm{u} \mathrm{p}$ to the calculated number of sample size was reached.

The examinations done included the followings:

a. Interview, to gather i nformation on personal characteristics and general aspects of the study subjects such as: age, sex, disease history including familial diseases, smoking, type of medication undertaken, etc, using prepared questionnaires.

b. Physical examinations, to obtain the information on blood pressure, temperature, weight, and other relevant physical features.

c. Laboratory examinations, $\mathrm{t}$ o obtain data of creatinine, homocysteine, ADMA, and $f$ asting glucose levels.
Data analysis was done using SPSS software for windows version 11.5 and Microsoft Excel 2000. OneSample Test was used to assess if data had normal distribution at a confidence limit $p=0.05$. Mean differential test of two independent groups was done using t test or Mann-Whitney test. Mean differential test of more than two groups was done by KruskalWallis $\mathrm{H}$ test. Linear correlation was assessed by the Pearson or Spearman. Non-linear correlation test was performed to assess correlation of two data groups: quadratically, logaritmatically or inversely.

\section{Results}

Based on data of the interviews, physical examinations, biochemicalexaminations, theindividualcharacteristics of the study subjects could be summarized as shown in Table 1.

\section{Table 1. Criteria for Kidney Dysfunction}

\begin{tabular}{l|ll} 
Stage & \multicolumn{1}{c}{ Description } & LFG $\left(\mathbf{m L} / \mathbf{m i n} / 1.73 \mathbf{~ m}^{2}\right.$ LPT) \\
\hline 1 & $\begin{array}{l}\text { Kidney defect with normal or } \\
\text { ncreased kidney function }\end{array}$ & $\geq 90$ \\
\hline $\begin{array}{l}\text { Kidney defect with mild decrease of } \\
\text { idney function }\end{array}$ & $60-89$ \\
3 & Moderate decrease of kidney function & $30-59$ \\
4 & Severe decrease of kidney function & $15-29$ \\
5 & Kidney failure & $<15$ (or dialysis)
\end{tabular}


Table 2. Characteristics of the study subject $(n=75)$

\begin{tabular}{|l|l|c|}
\hline Variable & Mean & Standard Deviation \\
\hline $\mathrm{n}=75$ & & \\
\hline Age $(\mathrm{yr})$ & 55 & 11.4 \\
\hline Fasting blood glucose $(\mathrm{mg} / \mathrm{dL})$ & 116.17 & 54.674 \\
\hline Cholesterol HDL $(\mathrm{mg} / \mathrm{dL})$ & 34.76 & 14.472 \\
\hline Triglyceride $(\mathrm{mg} / \mathrm{dL})$ & 145.67 & 76.775 \\
\hline tHcy $(\mu \mathrm{mol} / \mathrm{L})$ & 13.041 & 6.9006 \\
\hline Creatinine $(\mathrm{mg} / \mathrm{dL})$ & 2.6160 & 4.72629 \\
\hline LFG $\left(\mathrm{ml} / \mathrm{mnt}, 1.73 \mathrm{~m}^{2}\right)$ & 48.84 & 27.517 \\
\hline ADMA $(\mu$ mol/L) & 0.7329 & 0.25136 \\
\hline Systolic blood pressure $(\mathrm{mmHg})$ & 134.87 & 28.717 \\
\hline Diastolic blood pressure $(\mathrm{mmHg})$ & 83.68 & 15.086 \\
\hline
\end{tabular}

Note: LFG estimated by Cockroft-Gault formula

Table 3 indicates that there is a difference of the mean total homocysteine concentrations (tHcy) according to different severity levels of CKD, in which at higher stage of CKD there was more significant higher mean of tHcy concentration as compared with that at lower stage of CKD $(p=0.000)$.

Table 3. Analysis of tHcy concentrations ( $\mu \mathrm{mol} / \mathrm{L})$ according to CKD severity level

\begin{tabular}{l|l|l|l|l|l|l}
\hline CKD level & $\mathbf{n}$ & Mean & $\begin{array}{l}\text { Standard } \\
\text { deviation }\end{array}$ & Minimum & Maximum & $\mathbf{p}$ \\
\hline CKD stage 2 & 31 & 9.065 & 2.7212 & 5.3 & 17.7 & \\
CKD stage 3 & 23 & 11.965 & 3.9098 & 8.0 & 24.0 \\
CKD stage 4 & 10 & 16.620 & 4.6007 & 9.2 & 23.9 & \\
CKD stage 5 & 11 & 23.245 & 9.9221 & 9.7 & 38.9 & 0.000 \\
\hline
\end{tabular}

Note: stage 2 LFG value > 60; stage 3 LFG value 30-59; stage 4 LFG value 15-29; stage 5 LFG value $<15$; $p=$ probability * significance $(p<0.05)$

Analysis with least significance difference among the CKD stages showed a significant difference in tHcy concentration mean of each stage (stage 2 vs $3, p=0.036 ; 2$ vs $4, p=0.000 ; 2$ vs $5, p=0.000 ; 3$ vs $4, p=0.015 ; 3$ vs 5 $\mathrm{p}=0.000 ; 4$ vs $5 \mathrm{p}=0.003)$.

Table 4 indicates that at CKD stage 2 to 4 there was increasing tendency of insignificant ADMA concentration mean $(\mathrm{p}=0.210)$, however, ADMA concentration mean was lower at stage 5 . 
Table 4. ADMA concentrations ( $\mu \mathrm{mol} / \mathrm{L}$ ) based on CKD levels

\begin{tabular}{l|l|l|l|l|l|l|}
\hline CKD level & $\mathbf{n}$ & Mean & $\begin{array}{l}\text { Standard } \\
\text { deviation }\end{array}$ & Minimum & Maximum & $\mathbf{p}$ \\
\hline CKD stage 2 & 31 & 0.7001 & 0.19865 & 0.19 & 1.12 & \\
CKD stage 3 & 24 & 0.7521 & 0.24705 & 0.09 & 1.23 & 1.25 \\
CKD stage 4 & 10 & 0.8692 & 0.20770 & 0.60 & 1.09 & 0.210 \\
\hline CKD stage 5 & 11 & 0.6596 & 0.38416 & 0.10 & \\
\hline
\end{tabular}

Note: $p=$ probability

Tabel 5. Mean of homocysteine and ADMA concentration in DM and in non-DM patients

\begin{tabular}{l|c|c|c|}
\hline & Non DM & DM & p \\
\hline tHcy & $13.602 \pm 7.6987$ & $11.500 \pm 3.6878$ & 0.246 \\
\hline ADMA & $0.7525 \pm 0.2492$ & $0.6869 \pm 0.26103$ & 0.322 \\
\hline
\end{tabular}

Correlation between tHcy and ADMA concentration by linear regression showed that there was no significant correlation between ADMA concentration with tHcy with $\mathrm{r} 2=0.19$ and $\mathrm{p}=0.499$.

Correlation analysis between ADMA and tHcy concentration at each CKD level indicated the presence of positive correlation among them at CKD stage 2 and 3, however, inverse correlation between tHcy and ADMA concentration started to occur at stage 4

In this study there were $20 \mathrm{DM}$ patients and 55 non-DM patients. Higher tHcy and ADMA concentration means were observed but they were no significant difference between non-DM patients and DM patients.

If tHcy concentration $12 \mathrm{~mol} / \mathrm{L}$ was categorized as abnormal and this concentration represented cutoff value for PKV risk, by logistic binary test on the variables LFG, age, sex, presence of DM, hypertension and dyslipidemia, then it would show that LFG (estimation) is the most essential variable $(p=0.000)$ of the elevation of homocysteine concentration.

If ADMA concentration $>0.75 \mathrm{~mol} / \mathrm{L}$ was categorized as abnormal, this study showed that 33 subects (44\%) would have ADMA concentration $0.75 \mathrm{~mol} / \mathrm{L}$ and 42 subects $(5 \sqsubset \%)$ would have $>0.75$ $\mathrm{mol} / \mathrm{L}$. If ADMA concentration $>0.75 \mathrm{~mol} / \mathrm{L}$ was the cutoff value for PKV, with logistic binary test on the variables $\square \mathrm{GF} \square$ (estimation), age, sex, presence of DM, hypertension, and dyslipidemia, then it would show that sex and age were the statistically most significant variables ( $p 0.004$ and 0.012 ) of the elevation of ADMA concentration. $\square$ y sex variable, there were 32 female sub $\square$ ects $(42.7 \%)$ and 43 male sub $\square \operatorname{ect}(57.3 \%)$ involved in this study. $\square$ f the 32 female sub $\square$ ects, 24 sub $\square$ ects(75\%) were with ADMA concentration $>0.75$ mol/L (mean of age 55 years) and $\square$ sub $\square$ ects $(25 \%)$ with ADMA concentration $0.75 \mathrm{~mol} / \mathrm{L}$ (mean of age $\square$ years). Meanwhile, of 43 male sub $\square$ ectshere were $1 \square$ sub $\square$ ects (41.9\%) with ADMA concentration $>0.75 \mathrm{~mol} / \mathrm{L}$ (mean of age 52 years) and 25 sub $\square \operatorname{ects}(5 \square .1 \%$ ) with ADMA concentration $0.75 \mathrm{~mol} / \mathrm{L}$ (mean of age $5 \square$ years).

$\neg$ esult of the $t$ test showed there was no statistically significant difference between the age mean of the female study sub $\square$ ectswith elevated ADMA concentration and that of the female study sub $\square$ ects with non-elevated ADMA ( $\mathrm{p}=0.092)$. In contrast, it was found that the mean of age of the male study sub $\square$ ects with elevated ADMA concentration was significantly different from that of the male sub $\square$ ectshaving nonelevated ADMA concentration ( $\mathrm{p}=0.011$ ).

\section{Discussion}

able 3 indicated that in CKD patients there was a difference of the mean of tHcy concentration according to CKD severity levels, in which at later CKD stage, the mean of tHcy concentration was significantly higher $(p=0.000)$ than at earlier stage. 
CKD is almost often correlated with hyperhomosysteinemia. Plasma homocysteine concentration is closely correlated with decrease of LFG concentration in which elevated tHcy concentration starts to occur at LFG $<60 \mathrm{ml} /$ minute/1.73 m2. Prevalence of hyperhomocysteinemia in CKD is about $85-100 \%$ according to CKD severity. The relationship mechanism of LFG with elevated plasma homocysteine concentration is not yet fully understood (10).

In general, patients with kidney disease have normal methionine, betaine and vitamin B plasma level, increased AdotMet, Ado Hcy, cysteine and sulfate, and decreased serine concentration. It seems that in patients with kidney disease, the plasma homocysteine concentration has close correlation with plasma folate, AdoHcy and cysteine concentrations. The mechanism that can explain this phenomenon may be related with the inhibited homocysteine remethylation and impaired cysteine excretion

The mechanism of protein re-methylation inhibition is supported by results of studies that have indicated that successful treatment regimen could lower its concentration in CKD.

Impairment of cysteine excretion was reported by Suliman et al who suggested that in CKD inhibition of cysteinesulphunic acid decarboxylation, which is synthesis of cysteine and taurine alteration, caused elevation of hyperhomocysteine concentration.

A study using stable isotope method to observe systemic homocysteine metabolism in patients with kidney failure and in healthy individuals found that remethylation flux and total transmethylation were reduced in CKD patients.

Two mechanisms may explain the increase of plasma homocysteine concentration and reduced flux through methylation cycle in patients with kidney failure: 1) the presence of primary defect in homocysteine metabolism can result in homocysteine transulfuration impairment. On the other side, higher homocysteine concentration results in the slower methylation cycle by inhibiting transmethylation.; 2) the occurrence of pimary defect can result in homocysteine transmethylation. Of this study, the hypothesis was that an unknown substance - able to filtrate the glomerular sites- regulated homocysteine excretion through re-methylation or transulfuration.

Table 4 indicated that in CKD stage 2 to 4 there was a tendency of insignificant elevation of the mean ADMA concentration ( $\mathrm{p}=0.210$ ). In CKD stage 5 , there was lower ADMA concentration mean.

ADMA is arginine form modified post- translationally and synthesized by all cells during protein modification process. ADMA is well-known as an independent predictor for cardiovascular morbidity and mortality and its status as cardiovascular risk factor has been generally accepted. NOS competitive inhibition most likely becomes ADMA effect mechanism on the cardiovascular system, though nitric oxide (NO)-related mechanisms shouldn't be ignored. Only free ADMA, and not its bind protein, is able to inhibit NOS. Since NOS is an intracellular cell, concentration of free intracellular ADMA - not its plasma concentration - is associated in NOS inhibition. Free ADMA is formed exclusively through sequence of protein arginine residue methylation, followed by the protein proteolysis. There are two paths for ADMA clearance from cells. First, ADMA is degadraded enzymatically by DDAH to become citrulline and dimethylamine. Second, the path enhances ADMA transport from the cell to plasma through cationic acid transporters (CAT) in its plasma membrane. In addition, clearance from plasma compartment occurred through two routes: renal excretion and uptake by cell through CAT. Thus, CAT activity is involved in cellular release and ADMA uptake, and consequently, it plays important role in intracellular and inter-organ ADMA transportation. Both the kidney and liver seem to become important organs in ADMA clearance from plasma (9).

The actual mechanism that results in ADMA accumulation in CKD patients is not yet fully understood. One known mechanism is that reduced ADMA excretion has a linear relationship with reduced kidney function. In CKD, plasma ADMA concentration is increased to $1-3 \mathrm{~mol} / \mathrm{L}$ (11).

A study suggested that in intravenous dimethylarginine supplementation, $66 \%$ of filtrated SDMA would be reabsorbed, whereas only 5\% of filtrated ADMA would be reabsorbed.

Result of a study on molecular mechanism of increased ADMA plasma concentration in CKD using model mice with progressive CKD suggested that disregulation on enzyme PRMT and DDAH occurred as a result of oxidative stress. This mechanism was supported by the evidences that : (1) gene expression PRMT type I is increased by oxidized LDLin endothelial cells (EC) cultured by a redox-regulated mechanism; (2) DDAH activities in EC and smooth muscles is decreased in high-glucose condition, which is blocked by an antioxidant, superoxide dismutase. It is known that oxidative stress is increased in CKD patients. In addition to oxidative stress, there are uremic toxic 
like homocysteine and AGE products that decrease DDAH activity, which can cause disregulation of these enzyme.

The factors that have caused the insignificance of the ADMA elevation in our present study may possibly relate to the not-observed random variables like smoking, duration of hypertension, type of hypertension medication, and duration of diabetes and anti-diabetes therapy.

In this study the mean value of ADMA concentration was found to be lower in the earlier stages of CKD than in stage 5 . This will be explained in more detail in the next analysis of correlation of homocysteine and ADMA.

Result of the correlation analysis between tHcy and ADMA using linear regression showed that the data distribution among two variables indicated quadratic model, in which there was ADMA elevation simultaneously with tHcy up to the concentration of tHcy of about $20 \mathrm{~mol} / \mathrm{L}$, then decreased. The analysis found no significant correlation between ADMA and tHcy concentration.

Correlation analysis between ADMA and tHcy concentration in each CKD severity level indicated positive correlation in CKD stage 2 and 3, however, inverse correlation between tHcy and ADMA concentration started to appear in stage 4 .

Interaction between homocysteine and ADMA was estimated to occur by metabolic relationship between ADMA and transfer methyl reactions. ADMA is produced from methylated protein of arginin residue. PRMT type 1 utilized AdoMet=Sadenocylmethionine/SAM as a methyl donor and produced AdoHcy=S-adenocylhomocysteine $/ \mathrm{SAH}$ (and finally homocysteine) as its by product. Since ADMA is synthesized from proteolysis of methylated protein, the hypothesis was that increased SAMdependent proteinmethylation could resultinincreased ADMA production and increased homocysteine. This hypothesis was supported by an experimental study using animals (monkeys) supplemented with methionine-rich and folate-deficient food, the results of which indicated there was elevation of ADMA plasma concentration. Methionine-rich and folate-deficient food caused three times greater elevation of plasma tHcy and ADMA. It also suggested that the higher the ADMA concentration, the closer its correlation with tHcy; and occurrance vasodilation impairment was dependant on the endothelium of the carotid arteries of the animals (8).

In a clinical study, eight hours after supplementing standard oral methionine (100 mg $/ \mathrm{kg}$ ), plasma ADMA increased from $1.4 \quad 0.2$ to $2.0 \quad 0.2 \mathrm{M}(\mathrm{p}<0.05)$, plasma tHcy increased from 6.91 .6 to $28.43 .5 \mathrm{M}(\mathrm{p}<0.05)$, and brachial artery vasodilation decreased by $80 \%$. Brachial artery vasodilation had inverse correlation with plasma ADMA $(\mathrm{p}<0.05)$ and has linear correlation with L-arginine / ADMAratio $(\mathrm{p}<0.01)$. Similar findings were reported by Stuhlinger et al (8).

Evidence of the metabolic correlation between homocysteine and ADMA was also reported from studies with cell culture. Incubation of endothelial cells with L-methionine or L-homocysteine produced dosage-dependant ADMA elevations on the culture media (8).

Several control studies have reported the relationship between hyperhomocysteinemia and plasma ADMA elevation in human subjects with coronary artery disease, peripheral arterial disease, and stroke (8).

It wasn't fully understood how ADMA increased in hyperhomocysteinemia. Initially, it was hypothesized that the conditions that could cause homocysteine elevation included methionine-rich food, which could elevate SAM intracellular level and thus increase ADMA production by supporting SAMdependant PRMT activities. However, currently this mechanism is considered not likely, since typically hyperhomocysteinemia causes elevation of SAH level in tissue, but it is not true with the level of SAM . Although hyperhomocysteinemia probably does not increase PRMT activity with the increase of cellular SAMlevel, however, it is still possible that up regulation of PRMT expression occurs in hyperhomocysteinemia (8).

Other possible mechanism of ADMA elevation in hyperhomocysteinemia is decreased kidney function and decreased DDAH activity- that metabolize ADMA to citrulline. Homocysteine is reported to be able to inhibit DDAH activity in vitro, perhaps through an oxidative reaction with its active site residue (8).

In a study on mice with deficient-cystathionine -synthase (CBS) that resulted in high homocysteine, there was decreased mRNA concentration from two main DDAH enzymes: DDAH-1 and DDAH-2. This indicated that homocysteine could regulate DDAH expression and activity (8).

All recent studies on cell cultures in animals and human subjects reported that ADMA was metabolically associated with homocysteine. This indicated the influence of ADMA elevation on vascular dysfunction during hyperhomocystinemia. Homocysteine could 
increase ADMA by inhibiting DDAH activity or by stimulating oxidative stress which in turn wouldl inactivate DDAH oxidative. In contrast, homocysteine could elevate ADMA by stimulating endoplasmic reticulum stress and apoptosis that resulted in elevation of protein proteolysis containing methylarginine residue.

Correlation analysis between ADMA and tHcy concentration in each CKDseverity level indicated a positive correlation in CKD stage 2 and 3, however, inverse correlation between tHcy and ADMA concentration started to appear in CKD stage 4. This can be explained that in situation in which homocysteine elevated, SAH intracellular level could increase. Since SAH is a potent inhibitor for transmethylation reactions, its accumulation can result in macromolecules hypomethylation.

Consistent with this mechanism, DNA hypomethylation is observed on hyperhomocysteinemic patients with kidney problems. It was also observed that ADMA production by endothelial cells was inhibited by SAH incubation (9).

Appropriateness of an assay method is very important since it affects the correlation power between ADMA and homocysteine. In our study, ADMA was assayed by ELISA method, in which control precision among assays could be obtained (by coefficient of variation) by $14 \%$ of the control concentration range of $0.24-0.58$, all of which indicated that ADMA is closely metabolically associated with homocysteine. These evidences indicated a model in which ADMA elevation could be an integrating mechanism for vascular dysfunction in hyperhomocysteinemia. Homocysteine could increase ADMA by inhibiting DDAH activity through a direct chemical reaction with cysteine active site or by stimulating oxidative stress and DDAH oxidative inactivation as the result. On the other hand, homocysteine could elevate ADMA by stimulating endoplasmic reticulum stress and apoptosis that resulted in proteolysis elevation of protein containing methylarginine residue. ADMA accumulation in endothelial cells could inhibit eNOS to cause decreased NO products and endothelial dysfunction. Release of eNOS sequence triggered by ADMA or other oxidants like peroxinitrite could accelerate superoxide production and further decreased NO availability (8).

Result of the correlation analysis between ADMA and homocysteine showed no significant correlation between ADMA and tHcy concentration. This perhaps can be explained that in several studies conducted, some fundamental questions should yet to be answered concerning the pathophysiology of ADMA in hyperhomocysteinemia. Although most clinical studies have indicated that ADMA plasma increased in individuals with hyperhomosisteinemia, some of them have indicated that hyperhomosisteinemia wasn't always correlated with significant elevation of plasma ADMA. Similar differences were noted in animal models with hyperhomocysteinemia. There was three times greater elevation of plasma ADMA in hyperhomocysteinemic monkey, but only slight elevation occurred in plasma ADMA of CBS-deficient mice with hyperhomocysteinemia.

The correlation analysis between ADMA and tHcy concentration in each CKD severity level showed positive correlation between them in CKD stage 2 and 3, however, inverse correlation between tHcy and ADMA concentration started to appear in stage 4 . This could be explained that in situations in which homocysteine increased, intracellular SAH level could elevate. Since SAH is a potent inhibitor for transmethylation reactions, its accumulation could result in macromolecules hypomethylation. According to this mechanism, DNA hypomethylation is observed in hyperhomocysteinemic patients with end-stage kidney disease. Also observed that ADMA production by endothelial was inhibited by SAH-mediated incubation (9).

In this study, the assay on ADMA was performed by ELISA method, of which the control precision between assays (between day coefficient variation) was by $14 \%$ for control concentration range of $0.24-0.58$ $\mathrm{mol} / \mathrm{L}$ and $11 \%$ for control concentration range $0.6-$ $1.0 \mathrm{~mol} / \mathrm{L}$. This probably affected the test precision power that resuledt in error / weakening of correlation power between ADMA and homocysteine.

Teerlink in 2005 in his study reported that Pearson coefficient correlation distribution was obtained after 100 simulation experiments, thus to reveal that by increasing CV (coefficient correlation) in ADMA assay could resultin weakening of theestimation of correlation power between ADMA and homocysteine. Teerlink emphasized that with a very considerable number of study subjects, all those simulation experiments would still obtain very significant correlation. However, with less than 100 study subjects, its correlation would be statistically insignificant ( $\mathrm{p} \square 0.05$ ) in all simulation experiments, with $r \square 0.2$. In contrast with the crucial effect of CV elevation on the ADMA assay, artificial elevation of CV in homocysteine assay up to $10 \%$ most 
likely would not affect the correlation power.

In this study, there were $20 \mathrm{DM}$ patients and 55 nonDM patients studied. The correlation analysis found higher mean of tHcy and ADMA concentrations, but it was insignificant in the non-DM patients compared with than in the DM patients $(\mathrm{p}=0.246$ and $\mathrm{p}=0.322$ ). This probably was due to some variables being not observed in the study such as duration of diabetes and anti-diabetes medication as well as the fact that analysis was not carried out on each of the CKD stages in DM and non-DM groups.

If $\mathrm{tHcy}$ concentration $>12 \mathrm{~mol} / \mathrm{L}$ was categorized as abnormal and this concentration considered as cutoff value for CKD risk, by logistic binary test on the variables LFG, age, sex, presence of DM, hypertension and dyslipidemia, it was found that LFG (estimation) was the most important variable ( $p$ $=0.000)$ of homocysteine concentration elevation. This can be explained that homocysteine is mainly excreted by kidney while ADMA in only 5\%, thus LFG is very essential part of homocysteine concentration.

If ADMA concentration $>0.75 \mathrm{~mol} / \mathrm{L}$ (age mean 55 years) was categorized as abnormal (kit insert reagent), then from this study it was found 33 subjects $(44 \%)$ were with ADMA concentration $<0.75$ $\mathrm{mol} / \mathrm{L}$ and 42 subjects $(56 \%)>0.75 \mathrm{~mol} / \mathrm{L}$. If ADMA concentration $>0.75 \mathrm{~mol} / \mathrm{L}$ was considered cutoff value for CVD risk, by logistic binary test on variables GFR (estimation), age, sex, the presence of DM, hypertension, and dyslipidemia, it was found that sex and age were statistically the most important variables ( $p=0.004$ and 0.012$)$ of the elevation of ADMA.

Analysis on sex in this study showed 32 female subjects were with ADMA concentration $0.75 \mathrm{~mol} / \mathrm{L}$ (mean age 55 years) and 8 subjects $(25 \%)$ with ADMA concentration $<0.75 \mathrm{~mol} / \mathrm{L}$ (mean age 58 years). From $t$ test, it was found the age mean for the female subjects were with ADMA concentration that elevated insignificantly with the female subjects with nonelevated ADMA ( $\mathrm{p}=0.092)$.

Other result of the $t$ test showed that age means for male subjects with elevated ADMA were significantly different in male subjects with non-elevated ADMA $(p=0,011)$. This could be explained by several clinical studies which have reported convincingly of the role of ADMA in the progression of cardiovascular disease. In a cohort study on 25 patients with kidney failure, ADMA and age were found to be the strongest predictor for cardiovcascular events and total mortality, even after improvements on traditional and new risk factors had been made. Another study with control case design indicated that middle-age, non-smoking men with ADMA concentration in the highest quartile had experienced four times greater risk elevation in acute coronary events than other quartile (9).

Our present study revealed that $24(75 \%)$ of the female subjects had ADMA concentration elevation, while in the male subjects, ADMA elevation occurred in 18 of the male subjects (41.9\%). This was probably associated with the roleof women's hormone-especially estrogen - in suppressing ADMA concentration, in this study probably most of the female subjects were already in the menopause phase, thus their estrogen hormone had decreased and consequently elevated ADMA.

\section{Conclusion}

This study revealed that there was an increase in homocysteine and ADMA concentration according to CKD severity level. In CKD stage 2 to 4 there was a tendency of elevation of the ADMA concentration mean, but mean of ADMA concentration was lower in stage 5 . The positive correlation between homocysteine and ADMA concentration was showed by ADMA elevation concurrently with tHcy elevation up to 20 $\mathrm{mol} / \mathrm{L}$, and then decreased in stage 4 . $\square$ e also found out that there was negative correlation between tHcy and ADMA concentration that started to appear in stage 4. This can be explained that in situations in which homocysteine increases, intracellular $\square$ AHlevel can increase as well (hydrolysis reaction of $\square \mathrm{AH}$ to Hcy is reversible). $\square$ ince $\square \mathrm{AH}$ is a potent inhibitor for transmethylation, its accumulation can inhibit ADMA production by endothelial cells.

From these results, we suggest that a cohort study with equally distributed population number at every stage of CKD and a correlation study between hyperhomosisteinemia and ADMA, $\square$ RMT activity, $\mathrm{DDAH}$ and $\square \mathrm{O}$ formation than gold standard in endothelial dysfunction measurement of $\square$ ow mediated dilatation (FMD) should be conducted.

Thisstudybringsforward theopportunityoffinding or utilizing therapy target in hyperhomocysteinemic patients due to CKD in order to inhibit the effects of ADMA on endothelial dysfunction and progression of arteriosclerosis. 


\section{Acknowledgement:}

Thanks to Prof. Syakib Bakri MD. PhD, Wahidin Sudirohusodo Hospital, Makassar, who had helped this research.

\section{References:}

1. Bakri S. Deteksi Dini dan Upaya-upaya Pencegahan Progresifitas Penyakit Ginjal Kronik. Upacara Penerimaan Jabatan Guru Besar T etap dalam B idang IImu $P$ enyakit $D$ alam pada Fakultas Kedokteran. $\mathrm{H}$ asanuddin $\mathrm{U}$ niversity, $\mathrm{M}$ akassar. 2005.

2. Levin, A., et al. Chronic Kidney Disease Series : Cardiovascular Disease and The kidney Tracking A Killer in Chronic Kidney Disease. PostGraduate Medicine 2002, III (4).

3. C hade, A.R., Lerman, A., and Lerman, LO. K idney in Early Atherosclerosis. $H$ ypertension, 45:1042-1049. C ooke JP 2000, D oes ADMA Cause Endothelial Dysfunction?. Arterioscler Thromb Vasc Bio. 2005; 20 : 2032-2037.
4. Perna, A.F., Ingrosso, D., Satta, E. et al. Metabolic Consequences of Hyperhomocysteinemia in Uremia. Am J Kid Dis. 2001; 38(4) : S85-S90.

5. Vallance P, and Leipsi J. Cardiovascular Biology of the Asymmetric Dimethylarginine: D imethylarginine D imethylamino Hydrolase Pathway. Arterioscler Through Vasc Biol. 2004; 24:1023-1030.

6. Lin KY, and Lin SC. A Tale of Two Molecules: Nitric Oxide and Asymmetric Dimethylarginine. Acta Cardiol Sin. 2004; 20 : 201-211.

7. Dayal $S$ and Lentz SR. ADMA and Hyperhocysteinemia. Vasc Med. 2005; $10:$ S27-33.

8. Teerlink T. ADMA Metabolism and Clearance. Vas Med. 2005 ; $10:$ S73-81.

9. Guldener CV, Why is Homocysteine Elevated in Renal Failure And 68 What Can Be Expected From Homocysteine-Lowering?. Nephrol Dial Transplant. 2006; 21 : 1161-1166.

10. R avani, P., Tripepi G ., M alberti, F., Testa, S ., M allamaci, F., Zoccali, C. A $S$ immetrical $D$ imethylarginine $P$ redicts Progression to Dialysis and Death in Patients With Chronic Kidney Disease: A Competing Risks Modelling Approach. J Am Soc Neprol 2005;16 : 2449-2445.

Correction for Review Article - "Development of Immunopathobiogenesis on SIRS-Sepsis" by A. Guntur Hermawan (IBJ Vol. 1 No. 1 April 2009), on page 38. Figure3. Basic Concept of Sepsis (adpated from Guntur, 2000). We regret the error.

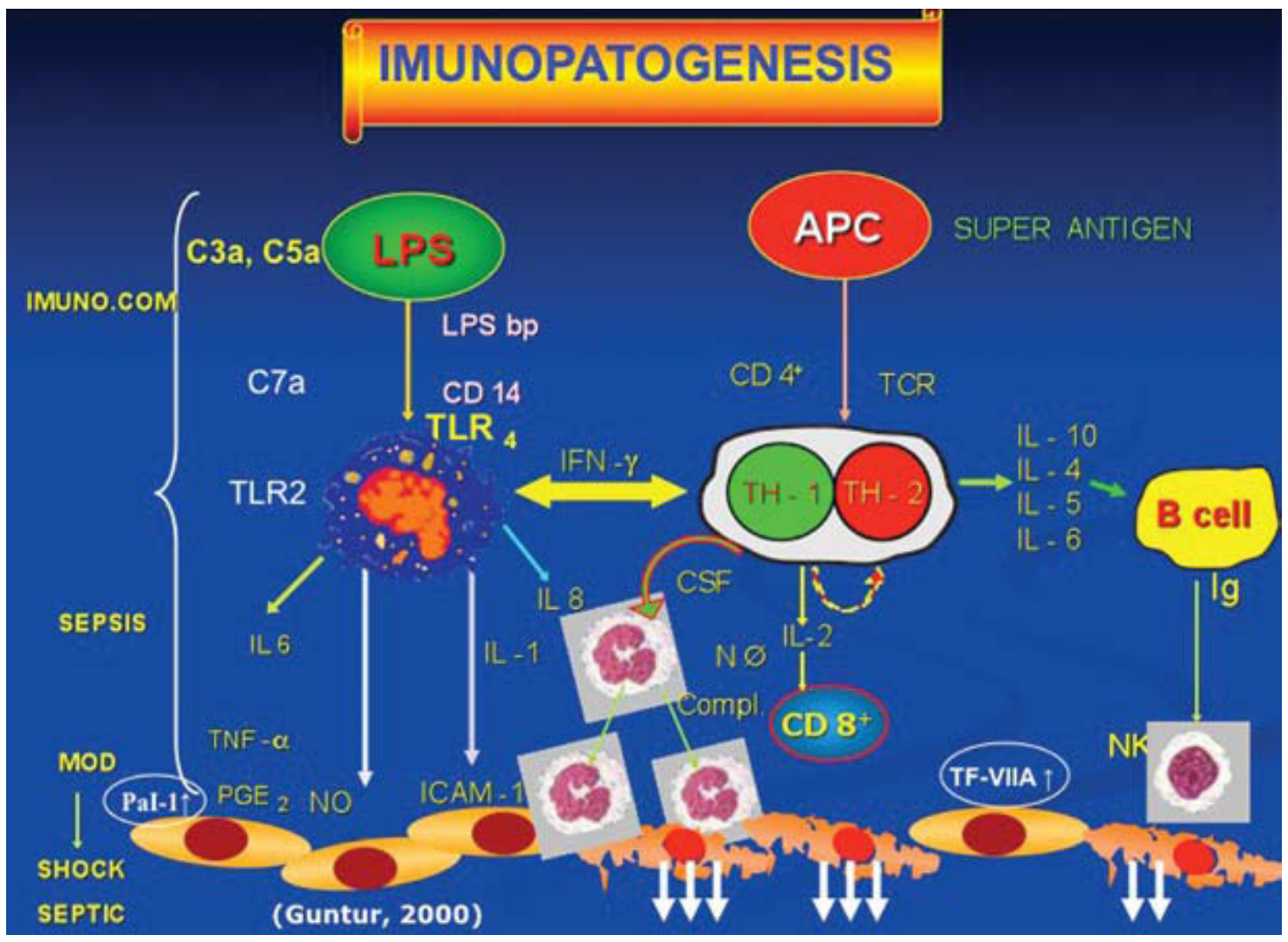

